\title{
Cortical Dysplasia in children
}

\author{
Helio Rubens Machado • Jorge Gonzalez-Martinez
}

Received: 3 July 2014 / Accepted: 8 July 2014

(C) Springer-Verlag Berlin Heidelberg 2014

Taylor and colleagues in 1971 published a seminal paper entitled Focal Dysplasia of the Cerebral Cortex in Epilepsy [1]. In this paper, they discussed in detail 10 cases of patients with epilepsy operated on by Murray Falconer ( 8 cases) in the Guy's-Maudsley-King's Neurosurgical Unit and 2 other cases treated at other Institutions. These cases were identified among 300 patients operated on during a period of 20 years at that Institution, and the neuropathological analysis was done by two eminent specialists (Bruton and Corsellis). Every case was exceptionally detailed both from the clinical and surgical point of view. Schematic drawings (see cover-illustration) clearly depicted the typical aspect of the cortex thickening and blurring whose image could only be seen preoperatively decades later after the introduction of magnetic resonance imaging into clinical practice. They also described at microscopic examination the characteristic " ...disruption of the normal cortical lamination and an excess of large aberrant neurons scattered randomly through all but the first layer... the most characteristic feature, therefore, was the disorganization both of the cortical architecture and of many of its individual neurons. In seven of 10 cases the anarchy was aggravated by the addition of malformed cells of uncertain origin with large, sometimes multiple, nuclei surrounded by an excess of opalescent pseudopodic cytoplasm" [1].

Although some authors had already published isolated cases of the same pathology, Taylor's work is an original

\section{H. R. Machado $(\triangle)$}

Center for Epilepsy Surgery, Department of Surgery and Anatomy, Ribeirao Preto Medical School, University of Sao Paulo,

Ribeirao Preto, Sao Paulo, Brazil

e-mail: hrmachad@fmrp.usp.br

J. Gonzalez-Martinez

Epilepsy Center, Neurological Institute, Cleveland Clinic, Cleveland, $\mathrm{OH}$, USA

e-mail: GONZALJ1@ccf.org contribution in the sense that he correctly stressed the main features of the disease, named it, and gave typical clinical features and image correlates. A quick search at PubMed using the terms Cortical Dysplasia (CD) and Epilepsy shows 3,041 papers to date with a steady increase in number of publications after 1995. It took almost a quarter of a century before we realized the impact of that publication in the field of pediatric epilepsy surgery.

$\mathrm{CD}$ represents the most common etiological entity concerning epilepsy surgical cases in children amounting roughly to a third of all cases. Its variegated mode of presentation and protean character from patient to patient justifies this publication aimed at pointing the major advances in the presentation at diagnosis, indications, and results of surgery. Epilepsy surgery remains most successful for "lesional" epilepsy, and removal of the lesion, with or without adjacent tissue resection is sufficient to permanently control the epileptic activity in some patients [2]. MRI anatomic and signal abnormalities have been described in $\mathrm{CD}$, but despite significant MRI abnormalities in most cases of CD, up to $25 \%$ of patients with "normal" MRIs had histopathologic changes in focal lesions. Consequently, a sizable number of patients with CD do not achieve a good outcome even after "complete" resection of the lesion. These results suggest that current imaging (and evaluation) techniques may not accurately map the extent of dysplastic and epileptogenic regions in CD. In other words, the topographic and functional relationships between MRI-revealed focal CD and the epileptogenic zone are far from clear and unequivocal: neither all focal $C D$ nor all components of a focal $\mathrm{CD}$ are necessarily epileptogenic. These clinical-radiological features make surgical treatment intriguing and challenging.

The main intention is to call the attention of the pediatric neurosurgeon non-devoted to epilepsy surgery that this entity, sometimes elusive, is common on a daily basis and he should integrate the team of specialists to address $\mathrm{CD}$ with specific 
tools if good long-term results are intended. CD should not be treated as brain tumor surgery as its non-clearly discernible limits may make the surgery unsuccessful and prolong suffering, sometimes adding sequelae to a child with heavy social and familial impact. We thank all contributors and dedicated centers that have given their contribution and expertise to this annual issue.

\section{References}

1. Taylor DC, Falconer MA, Bruton CJ, Corsellis JA (1971) Focal dysplasia of the cerebral cortex in epilepsy. J Neurol Neurosurg Psychiatry 34:369-387

2. Wyllie E, Comair YG, Kotagal P, Bulacio J, Bingaman W, Ruggieri P (1998) Seizure outcome after epilepsy surgery in children and adolescents. Ann Neurol 44:740-748 